\title{
CINÉTICAS DE OXIDAÇÃO E CÁLCULO DA ENERGIA DE ATIVAÇÃO APARENTE PARA FORMAÇÃO DOS ÓXIDOS EM AÇO INOXIDÁVEL AUSTENÍTICOS LAMINADO 253 MA A ALTAS TEMPERATURAS AO AR*
}

\author{
André de Albuquerque Vicente ${ }^{1}$ \\ Débora Arruda Cabral \\ lgor Jordão Marques ${ }^{3}$ \\ Tiago Felipe de Abreu Santos ${ }^{4}$ \\ Denise Crocce Romano Espinosa ${ }^{5}$ \\ Jorge Alberto Soares Tenório ${ }^{6}$
}

\begin{abstract}
Resumo
O estudo detalhado das cinéticas de oxidação a altas temperaturas ao ar permitiu o cálculo da energia de ativação aparente do processo de oxidação de um aço inoxidável austenítico laminado do tipo 22.12. Utilizou-se o aço inoxidável austenítico microligado ao cério (253 MA®) que é um aço projetado para trabalhos a altas temperaturas. Observou-se cinéticas de oxidação parabólicas em todas as temperaturas estudadas: $800,900,1000$ e $1100^{\circ} \mathrm{C}$. Os $\mathrm{K}_{\mathrm{ps}}$ encontrados neste trabalho reforçam a tese de que ocorra a formação de películas protetoras de $\mathrm{Cr}_{2} \mathrm{O}_{3}$ e $\mathrm{SiO}_{2}$, o que reforça a hipótese que as mesmas tenham sido efetivamente formadas após os estágios iniciais de oxidação.
\end{abstract}

Palavras-chave: Aço Inoxidável; 253 MA®; Oxidação a Altas Temperaturas; Cinética.

\section{KINETICS OF OXIDATION AND CALCULATION OF APPARENT ACTIVATION ENERGY FOR THE FORMATION OF OXIDES IN ROLLED STAINLESS STEEL 253 MA AT HIGH TEMPERATURES IN AIR}

\section{Abstract}

The detailed study of the kinetics of oxidation at high temperatures in the air allowed the calculation of the apparent activation energy of the oxidation process of a rolled austenitic stainless steel of type 22.12. The austenitic stainless steel microalloyed to cerium (253 MA®) that is a steel designed for works at high temperatures was used. Parabolic oxidation kinetics were observed at all temperatures studied: 800, 900, 1000 and $1100^{\circ} \mathrm{C}$. The $\mathrm{K}_{\mathrm{p}}$ found in this work reinforce the thesis that the formation of protective films of $\mathrm{Cr}_{2} \mathrm{O}_{3}$ and $\mathrm{SiO}_{2}$ occurs, reinforcing the hypothesis that they have been effectively formed after the initial stages of oxidation.

Keywords: Stainless Steel; 253 MA®; High Temperatures Oxidation; Kinetics.

\footnotetext{
Doutorando do Departamento de Engenharia Química da EPUSP.

Engenheranda do Departamento de Engenharia Metalúrgica e de Materiais da EPUSP.

Engenherando do Departamento de Engenharia Mecânica da UFPE.

Professor Adjunto do Departamento de Engenharia Mecânica da UFPE.

Professora Associada do Departamento de Engenharia Química da EPUSP.

Professor Titular do Departamento de Engenharia Química da EPUSP.
} 


\section{INTRODUÇÃO}

O estudo da oxidação a altas temperaturas de ligas é mais complexo que o da oxidação de metais puros. Isto ocorre devido a um ou mais fatores listados a seguir: [1]

- Os metais presentes na liga apresentam diferentes afinidades com o oxigênio. Por isso, observa-se diferentes energias livres de formação dos óxidos;

- A oxidação de ligas pode gerar óxidos ternários e/ou quaternários;

- $\quad$ Os óxidos formados podem formar soluções sólidas;

- Os diversos íons metálicos apresentam diferentes mobilidades nos óxidos formados:

- $\quad$ Os diferentes metais formadores da liga apresentam coeficientes de difusão diferentes na liga;

- $\quad$ A dissolução do oxigênio na liga pode resultar em oxidação seletiva de um ou mais elementos de liga (oxidação interna). [1]

O uso de constantes de crescimento parabólicas, é uma forma conveniente de comparar taxas de crescimento de películas de óxidos com comportamento aproximadamente parabólico. A figura 1 apresenta constantes de crescimento parabólicas de diversos óxidos para a reação direta com o oxigênio.

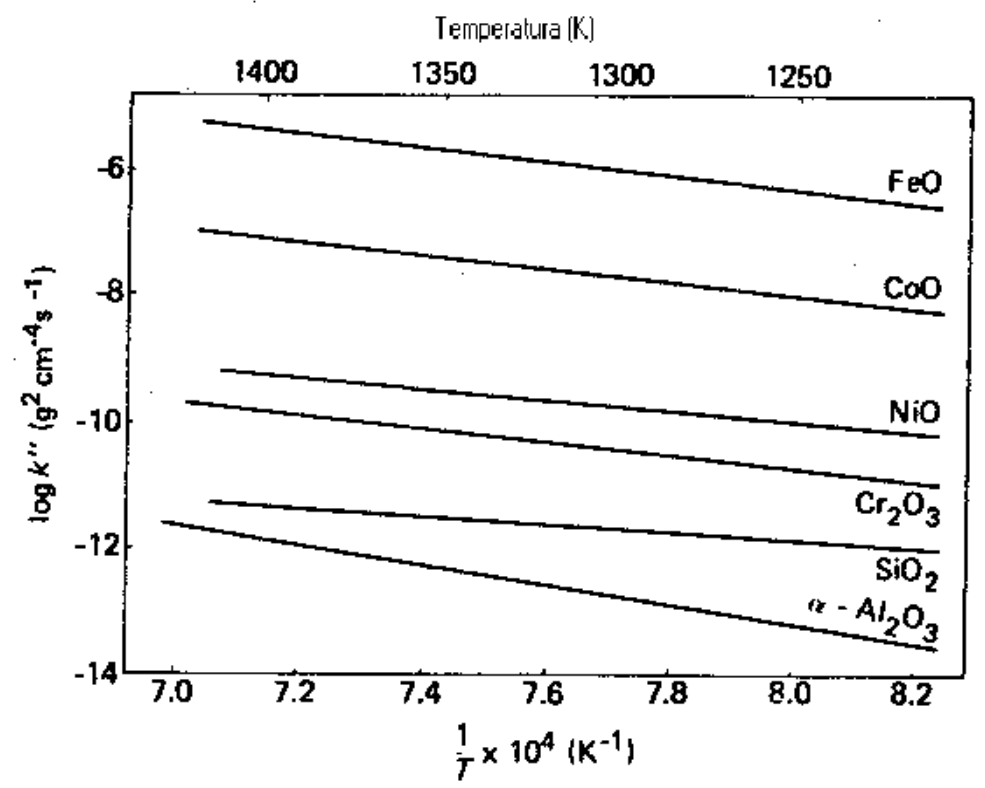

Figura 1 - Logarítmos das constantes de crescimento parabólicas de diversos óxidos em função da temperatura recíproca para a reação direta com o oxigênio. [1]

As taxas de crescimento de películas de óxidos não são controladas por suas propriedades termodinâmicas, mas sim pelos gradientes de concentração e pela mobilidade dos elementos envolvidos na reação. [2]

Os coeficientes de difusão para o oxigênio e para os íons metálicos através dos óxidos protetores, como é o caso da sílica e da cromita $\left(\mathrm{SiO}_{2}\right.$ e $\left.\mathrm{Cr}_{2} \mathrm{O}_{3}\right)$ são limitadores da velocidade de crescimento dos óxidos. [2]

$\mathrm{Na}$ oxidação de metais puros a altas temperaturas, a taxa de crescimento dos óxidos será controlada por difusão iônica através de defeitos na camada de óxido se algumas condições forem satisfeitas:

1. A camada é macroscopicamente densa;

2. A camada apresentar boa condutibilidade elétrica;

3. A camada apresentar aderência uniforme ao metal. 
Se estas condições forem observadas a taxa de oxidação do metal a altas temperaturas deverá ser parabólica. [3-12]

As estabilidades termodinâmicas de alguns óxidos são ilustradas na tabela 1.

Tabela 1 - Propriedades Termodinâmicas de Alguns Óxidos a $1000^{\circ} \mathrm{C}$ [13]

\begin{tabular}{|c|c|c|c|}
\hline $\begin{array}{l}\text { Elemento } \\
\text { Químico }\end{array}$ & $\begin{array}{l}\text { Óxido } \\
\text { Formado }\end{array}$ & $\begin{array}{l}\text { Energia Livre de Formação } \\
\text { (kJ/mole de } \mathrm{O}_{2} \text { reagido) }\end{array}$ & $\begin{array}{l}\mathrm{Log} \\
\mathrm{P}_{02}\end{array}$ \\
\hline $\mathrm{Ni}$ & $\mathrm{NiO}$ & -60.0 & -10.3 \\
\hline Co & $\mathrm{CoO}$ & -69.3 & -11.9 \\
\hline $\mathbf{W}$ & $\mathrm{WO}_{2}$ & -85.7 & -14.7 \\
\hline $\mathbf{F e}$ & $\mathrm{FeO}$ & -86.5 & -14.8 \\
\hline Mo & $\mathrm{MoO}_{2}$ & -86.6 & -14.9 \\
\hline $\mathrm{Cr}$ & $\mathrm{Cr}_{2} \mathrm{O}_{3}$ & -127 & -21.8 \\
\hline Mn & MnO & -139 & -23.9 \\
\hline $\mathrm{Ta}$ & $\mathrm{Ta}_{2} \mathrm{O}_{5}$ & -142 & -24.2 \\
\hline $\mathrm{Nb}$ & $\mathrm{CbO}$ & -146 & -25.1 \\
\hline Si & $\mathrm{SiO}_{2}$ & -163 & -28.0 \\
\hline $\mathrm{Ti}$ & TiO & -190 & -32.7 \\
\hline Al & $\mathrm{Al}_{2} \mathrm{O}_{3}$ & -202 & -34.7 \\
\hline
\end{tabular}

Obs: $P_{02}=$ Pressão de dissociação do óxido

É importante frisar que o crescimento a alta temperatura das películas de óxidos têm apresentado comportamentos parabólicos em diversos trabalhos anteriores. [3-12]

\section{MATERIAIS E MÉTODOS}

Para a preparação dos corpos de prova para os ensaios de oxidação ao ar a altas temperaturas utilizando-se equipamento de corte da marca Isomet, modelo Precision Saw 1000, e disco de diamante foi possível obter corpos de prova de $10 \mathrm{~mm}$ de largura, $10 \mathrm{~mm}$ de comprimento e espessura de $1 \mathrm{~mm}$.

As amostras foram furadas utilizando-se uma furadeira de bancada de marca Dremel, modelo 395 tipo 5 , e brocas de $1 \mathrm{~mm}$ de diâmetro, para poder fixa-las à haste da balança termogravimétrica.

Empregou-se o polimento manual convencional utilizando-se lixas d'água (180, 220, 320,400 e 600 mesh) com o objetivo de se padronizar o acabamento superficial das amostras.

As espessuras e dimensões das amostras foram determinadas com o auxílio de um micrômetro. As áreas de todas as amostras foram medidas antes do respectivo ensaio de oxidação para determinar o ganho de massa por unidade de área. Antes do início dos ensaios de oxidação, todas as amostras foram limpas com álcool e, posteriormente, com acetona em aparelho de ultrassom.

Esta etapa da preparação é muito importante pois é uma forma de padronizar-se o acabamento superficial das amostras retirando resíduos de fluido de corte e/ou das lixas.

Os ensaios de oxidação foram realizados utilizando-se balança termogravimétrica da marca Setaram do LAT-LAREX USP. Foram realizados ensaios isotérmicos ao ar nas temperaturas $800,900,1000,1100 \stackrel{\circ}{ } \mathrm{C}$.

Todos os ensaios foram realizados ao ar. O tempo de duração dos ensaios é de 24 horas. 


\section{RESULTADOS E DISCUSSÃO}

A figura 2 apresenta as curvas de ganho de massa obtidas para a amostra do aço inoxidável austenítico laminado (253 MA®) para ensaios isotérmicos de oxidação ao ar nas temperaturas estudadas, de 800 até $1100{ }^{\circ} \mathrm{C}$.

\section{MA}

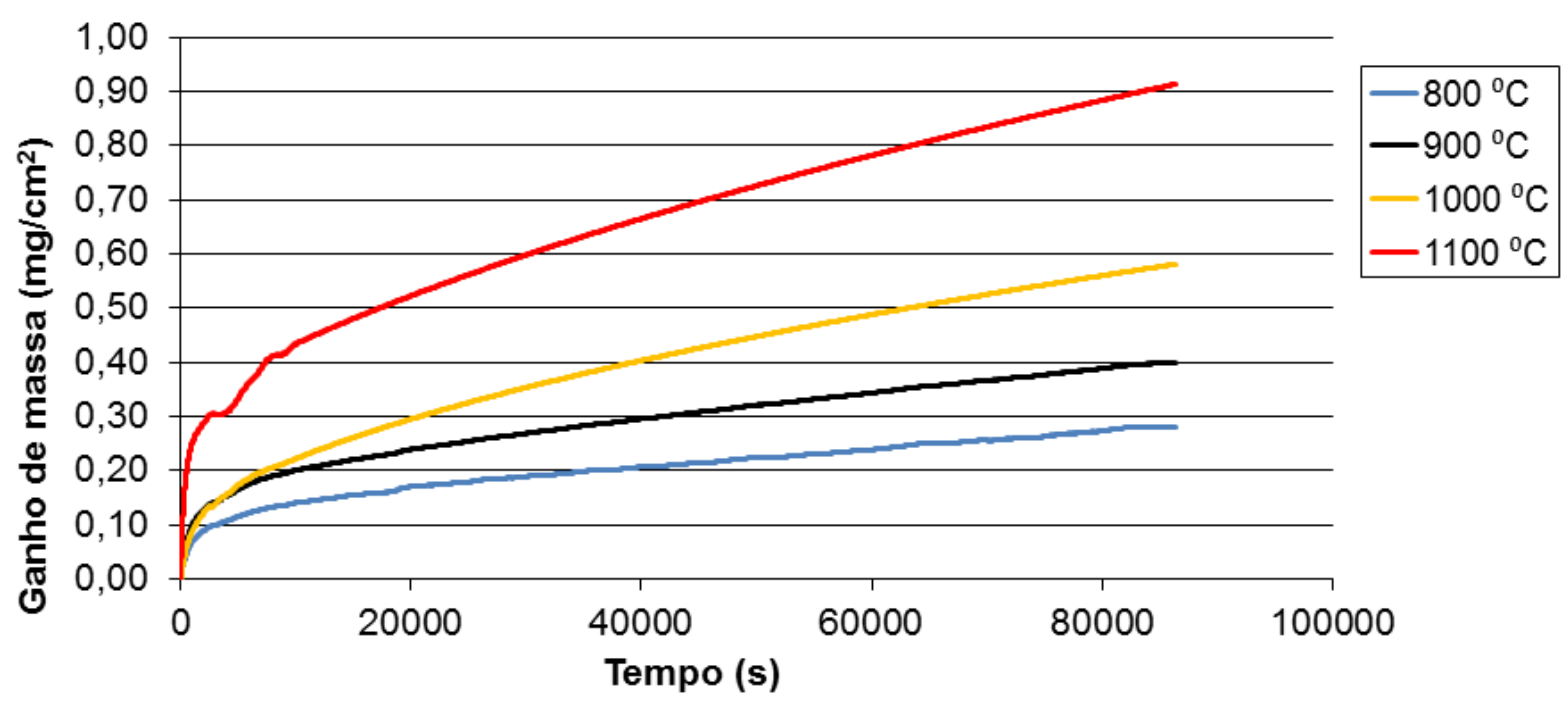

Figura 2. Ensaios de oxidação ao ar do aço inoxidável austenítico laminado (253 MA®)

$\mathrm{Na}$ figura 2 observa-se aumento das cinéticas de oxidação com o aumento da temperatura de ensaio de 800 até $1100^{\circ} \mathrm{C}$.

Foram testados os comportamentos parabólico, exponencial e linear para as cinéticas de crescimento dos óxidos formados.

A análise dos ensaios termogravimétricos do aço inoxidável austenítico laminado (253 MA®) mostrou uma cinética de oxidação complexa, passando de logarítmica nos instantes iniciais para um posterior comportamento parabólico.

O aço inoxidável austenítico laminado (253 $\mathrm{MA} \AA$ ) apresentou comportamento parecido quanto ao mecanismo de crescimento das películas de óxidos nos ensaios de oxidação isotérmicos ao ar nas temperaturas $800,900,1000$ e $1100{ }^{\circ} \mathrm{C}$.

A tabela 2 mostra os coeficientes das regressões $\left(R^{2}\right)$ para os comportamentos parabólico, linear e logarítmico dos ensaios de oxidação ao ar a altas temperaturas do aço inoxidável austenítico laminado (253 MA®).

Tabela 2 - Coeficientes das regressões $\left(\mathrm{R}^{2}\right)$ para os comportamentos parabólico, linear e logarítmico dos ensaios de oxidação ao ar a altas temperaturas

\begin{tabular}{|c|c|c|c|}
\hline Temperatura $\left({ }^{\circ} \mathbf{C}\right)$ & Parabólico & Linear & Logarítmico \\
\hline $\mathbf{8 0 0}$ & 0,92 & 0,34 & 0,92 \\
\hline $\mathbf{9 0 0}$ & 0,92 & 0,34 & 0,92 \\
\hline $\mathbf{1 0 0 0}$ & 0,99 & 0,48 & 0,90 \\
\hline $\mathbf{1 1 0 0}$ & 0,95 & 0,10 & 0,90 \\
\hline
\end{tabular}

Pode-se observar na tabela 2 que os comportamentos parabólico e logarítimico são os que apresentam os melhores valores para os coeficientes das regressões $\left(R^{2}\right)$ quando analisa-se o ensaio completo. 
$\mathrm{Na}$ oxidação a alta temperatura de aços inoxidáveis austeníticos, o rápido crescimento inicial dos óxidos de ferro contribui para uma alta taxa inicial de crescimento não parabólica e ao mesmo tempo a camada de $\mathrm{Cr}_{2} \mathrm{O}_{3} \mathrm{eSiO}_{2}$ vai sendo formada a uma baixa taxa de crescimento parabólica.

Devido ao às grandes velocidades de oxidação observadas nas etapas iniciais da formação dos óxidos, dividiu-se a curva de ganho de massa em função do tempo em dois períodos. Para tempos inferiores a 10.000 segundos definiu-se o período inicial de oxidação.

A tabela 3 mostra os coeficientes das regressões $\left(R^{2}\right)$ para os comportamentos parabólico e logarítmico dos ensaios de oxidação ao ar do aço inoxidável austenítico laminado (253 MA®) quando analisadas as etapas iniciais de crescimento da película de óxido.

Tabela 3 - Coeficientes das regressões $\left(R^{2}\right)$ para os comportamentos parabólico e logarítmico nas etapas iniciais de crescimento da película de óxido dos ensaios de oxidação ao ar.

\begin{tabular}{|c|c|c|}
\hline Temperatura $\left({ }^{\circ} \mathrm{C}\right)$ & Parabólico & Logarítmico \\
\hline 800 & 0,84 & 0,98 \\
\hline 900 & 0,83 & 0,98 \\
\hline 1000 & 0,72 & 0,98 \\
\hline 1100 & 0,77 & 0,98 \\
\hline
\end{tabular}

Pode-se observar na tabela 3 que o comportamento logarítmico é o que apresenta os melhores valores para os coeficientes das regressões $\left(R^{2}\right)$ nas etapas iniciais de crescimento das películas de óxido, o que sugere a formação de óxidos de ferro $\left(\mathrm{Fe}_{2} \mathrm{O}_{3}, \mathrm{Fe}_{3} \mathrm{O}_{4}\right.$ e $\mathrm{FeO}$ ) a uma alta taxa inicial de crescimento.

A tabela 4 mostra os coeficientes das regressões $\left(R^{2}\right)$ para os comportamentos parabólico e logarítmico dos ensaios de oxidação ao ar do aço inoxidável austenítico laminado (253 MA®) para tempos acima de 10000 segundos.

Tabela 4 - Coeficientes das regressões $\left(R^{2}\right)$ para os comportamentos parabólico e logarítmico para tempos acima de 10000 segundos dos ensaios de oxidação ao ar.

\begin{tabular}{|c|c|c|}
\hline Temperatura $\left({ }^{\circ} \mathrm{C}\right)$ & Parabólico & Logarítmico \\
\hline 800 & 1,00 & 0,96 \\
\hline 900 & 1,00 & 0,97 \\
\hline 1000 & 1,00 & 0,98 \\
\hline 1100 & 1,00 & 0,97 \\
\hline
\end{tabular}

Observa-se na tabela 4 que o comportamento parabólico apresenta os melhores valores para os coeficientes das regressões $\left(R^{2}\right)$. Na literatura existem várias citações sobre o comportamento parabólico da cinética de oxidação de ligas resistentes à corrosão a alta temperatura. [3-12]

Para o levantamento das constantes parabólicas de crescimento $\left(K_{p},\right)$ elevou-se os valores de ganho de massa ao quadrado para tempos acima de 10000 segundos e efetuou-se regressão linear dos valores de ganho de massa ao quadrado em função do tempo. Desprezou-se as etapas iniciais de oxidação.

A figura 3 apresenta as regressões lineares das cinéticas de reações parabólicas do aço inoxidável austenítico laminado (253 MA®) nas diversas temperaturas de ensaios isotérmicos de oxidação ao ar para tempos acima de 10000 segundos. 
a)

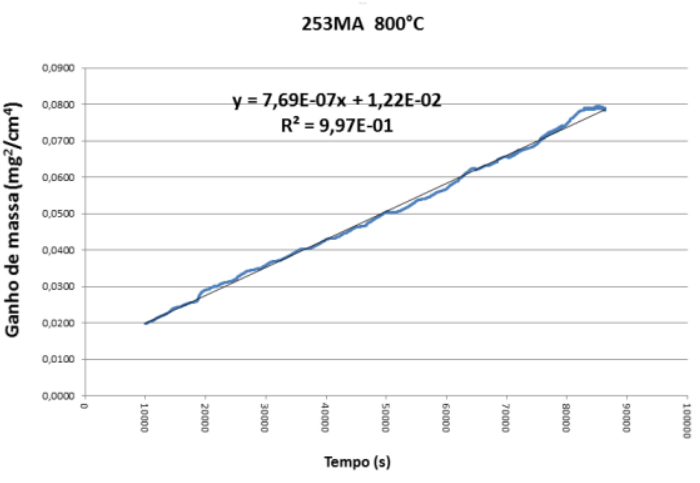

253MA $1000^{\circ} \mathrm{C}$

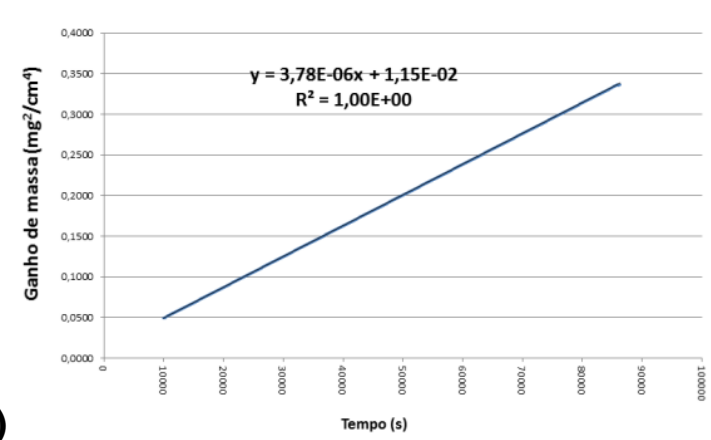

253MA $900^{\circ} \mathrm{C}$

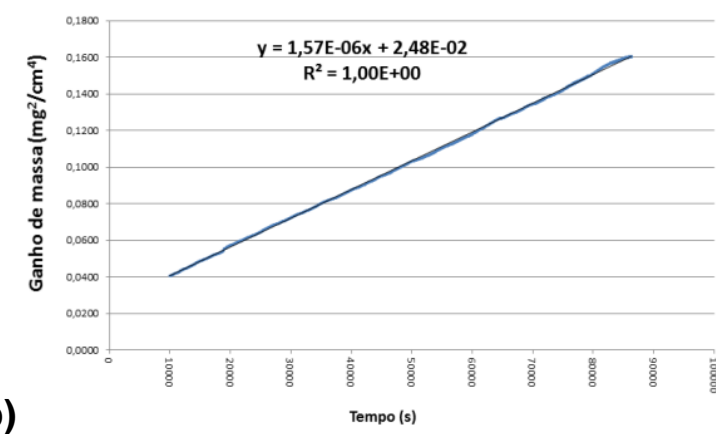

$253 \mathrm{MA} 1100^{\circ} \mathrm{C}$

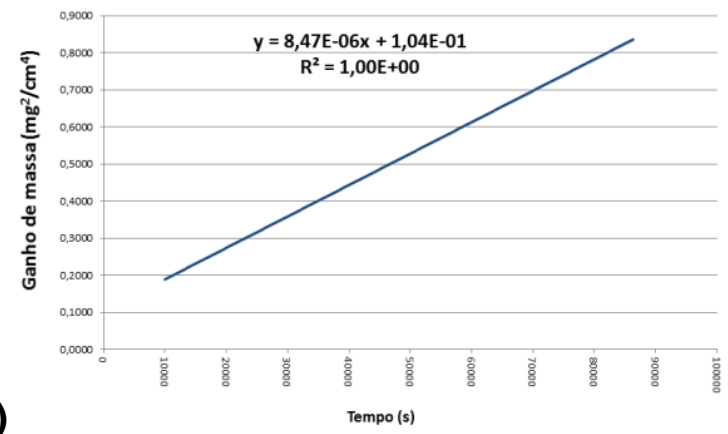

Figura 3 - Regressões lineares das cinéticas de reação parabólicas nas diversas temperaturas de ensaios isotérmicos de oxidação ao ar para tempos acima de 10000 segundos.

a) $800{ }^{\circ} \mathrm{C}$, b) $900^{\circ} \mathrm{C}$, c) $1000^{\circ} \mathrm{C}$ e d) $1100^{\circ} \mathrm{C}$

A tabela 5 mostra os valores das constantes parabólicas de crescimento $K_{p}$ e dos coeficientes das regressões $\left(R^{2}\right)$ para os comportamentos parabólicos dos ensaios de oxidação ao ar do aço inoxidável austenítico laminado (253 MA®) para tempos acima de 10000 segundos.

Tabela 5 - Constantes parabólicas de crescimento $\left(K_{p}\right)$ e coeficientes das regressões $\left(R^{2}\right)$ para o comportamento parabólico dos ensaios de oxidação ao ar para tempos acima de 10000 segundos

\begin{tabular}{|c|c|c|}
\hline Temperatura $\left({ }^{\circ} \mathbf{C}\right)$ & $\mathbf{K p}\left(\mathbf{m g}^{2} . \mathbf{c m}^{-4} . \mathbf{s}^{-1}\right)$ & $\mathbf{R}^{\mathbf{2}}$ \\
\hline 800 & $\mathbf{7 , 6 9 \times 1 0 - 7}$ & $\mathbf{1 , 0 0}$ \\
\hline 900 & $1,57 \times 10-6$ & 1,00 \\
\hline 1000 & $3,78 \times 10-6$ & 1,00 \\
\hline 1100 & $\mathbf{8 , 4 7 \times 1 0 - 6}$ & 1,00 \\
\hline
\end{tabular}

A figura 4 mostra os valores dos logarítmos de $K_{p}$ do aço inoxidável austenítico laminado (253 MA®) em função da temperatura recíproca para tempos acima de 10000 segundos. 
253 MA

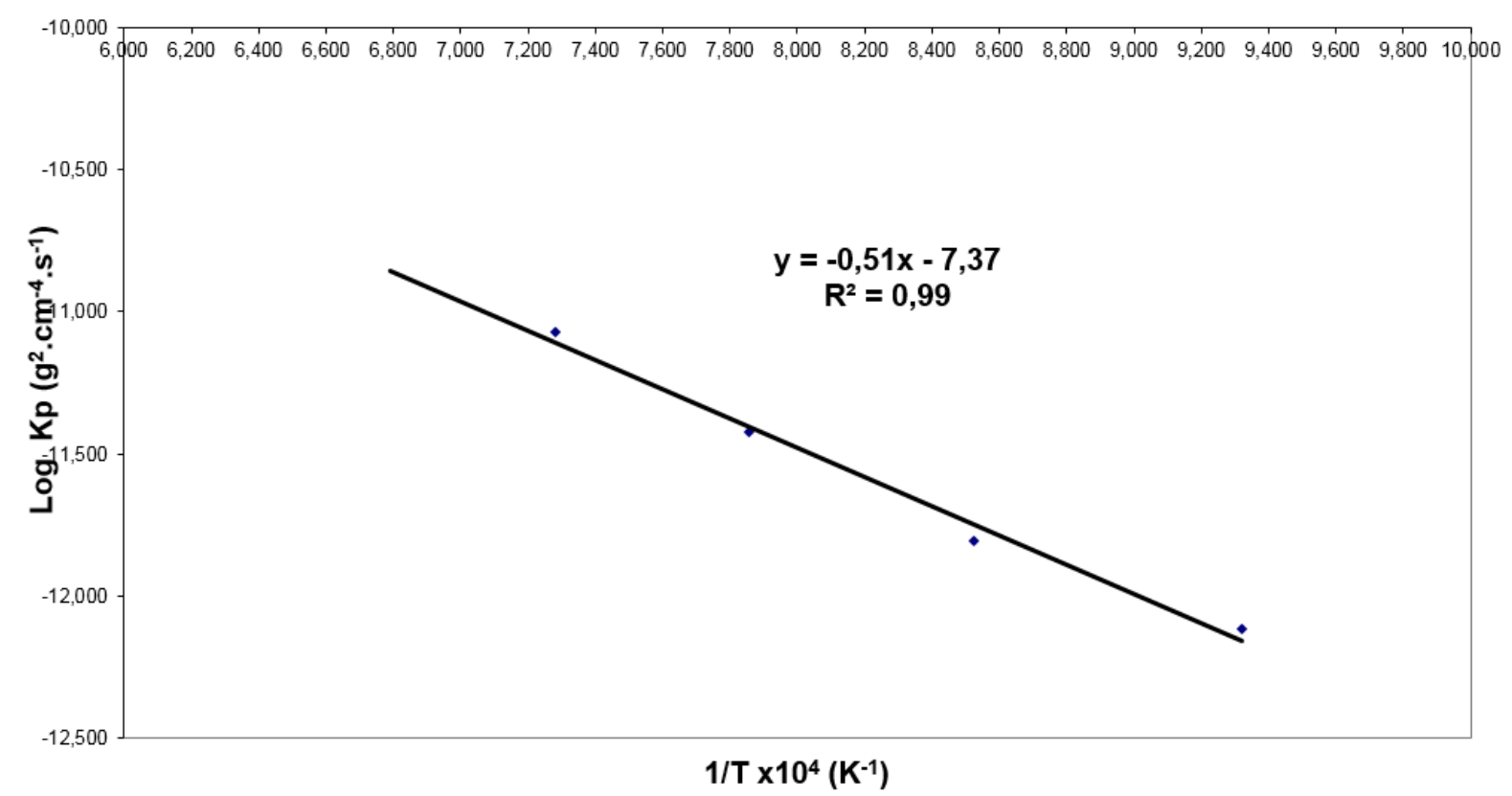

Figura 4 - Valores do log $\left(K_{p}\right)$ do aço inoxidável austenítico laminado (253 MA®) em função da temperatura recíproca para tempos acima de 10000 segundos.

O valor de $K_{p}$ é dado pela equação 1 , uma vez que trata-se de um processo ativado termicamente.

$$
K_{p}=K_{0} \exp \left(\frac{-Q}{R T}\right) \quad \text { (Equação 1) [10] }
$$

$Q$ é a energia de ativação para o processo de difusão e $K_{0}$ é uma constante. Os valores de $Q$ e $K_{0}$ podem ser determinados a partir do gráfico de $\log \left(K_{p}\right)$ versus $1 / T$. $\mathrm{Na}$ figura 4 plotou-se os logarítmos de $\mathrm{K}_{\mathrm{p}}$ em função da temperatura recíproca para do aço inoxidável austenítico laminado (253 MA®), obtendo-se a equação 2:

$$
\log \left(K_{p}\right)=\left[-0,51 \times 10^{4}\left(\frac{1}{T}\right)-7,37\right] \quad(\text { Equação 2) }
$$

A tabela 6 apresenta os valores das constantes parabólicas de crescimento nas temperaturas de ensaio de oxidação ao ar de 800 a $1100^{\circ} \mathrm{C}$ calculados utilizando-se a equação 2 .

Tabela 6 - Constantes parabólicas de crescimento $K_{p}$ do aço inoxidável austenítico laminado (253 $\mathrm{MA}(\mathrm{B})$ para as temperaturas de 800 a $1100^{\circ} \mathrm{C}$ calculadas através da equação 2.

\begin{tabular}{|c|c|}
\hline Temperatura $\left({ }^{\circ} \mathbf{C}\right)$ & $\mathbf{K}_{\mathbf{p}}\left(\mathbf{g}^{2} / \mathbf{c m}^{4} . \mathbf{s}\right)$ \\
\hline 800 & $\mathbf{7 , 5 3 \times 1 0 ^ { - 1 3 }}$ \\
\hline 900 & $\mathbf{1 , 9 2 \times 1 0 ^ { - 1 2 }}$ \\
\hline 1000 & $\mathbf{4 , 2 0 \times 1 0 ^ { - 1 2 }}$ \\
\hline 1100 & $\mathbf{8 , 2 3 \times 1 0 ^ { - 1 2 }}$ \\
\hline
\end{tabular}

A equação 3 baseia-se na equação 1 , fornecendo $o$ valor da energia de ativação aparente para o processo de oxidação a altas temperaturas. 


$$
E_{a}=\frac{2,303 \times R}{\left(\frac{1}{T_{2}}-\frac{1}{T_{1}}\right)} \times \log \left(\frac{K_{1}}{K_{2}}\right) \quad(\text { Equação 3) }
$$

$\mathrm{R}$ é a constante universal dos gases $\left(8,314 \mathrm{~J} \cdot \mathrm{mol}^{-1} \cdot \mathrm{K}^{-1}\right), \mathrm{K}_{1}$ e $\mathrm{K}_{2}$ são as constantes parabólicas de crescimento nas temperaturas $\mathrm{T}_{1} \mathrm{e} \mathrm{T}_{2}$, respectivamente.

Aplicando-se os valores da tabela 6 na equação 3, obtém-se a energia de ativação aparente para o processo de oxidação a alta temperatura do aço inoxidável austenítico laminado (253 MA®).

$$
E_{a}=\frac{2,303 \times 8,314}{\left(\frac{1}{1373}-\frac{1}{1073}\right)} \times \log \left(\frac{7,53 \times 10^{-13}}{8,23 \times 10^{-12}}\right) \cong 97,66 \mathrm{~kJ}
$$

A figura 5 mostra que os valores de $\mathrm{K}_{\mathrm{p}}$ s encontrados neste trabalho estão de acordo com os observados na figura 1 quando há formação de películas protetoras de $\mathrm{Cr}_{2} \mathrm{O}_{3}$ e $\mathrm{SiO}_{2}$, o que reforça a hipótese que as mesmas tenham sido efetivamente formadas após os estágios iniciais de oxidação.

\section{$\log \left(K_{p}\right)$ em função da temperatura recíproca}

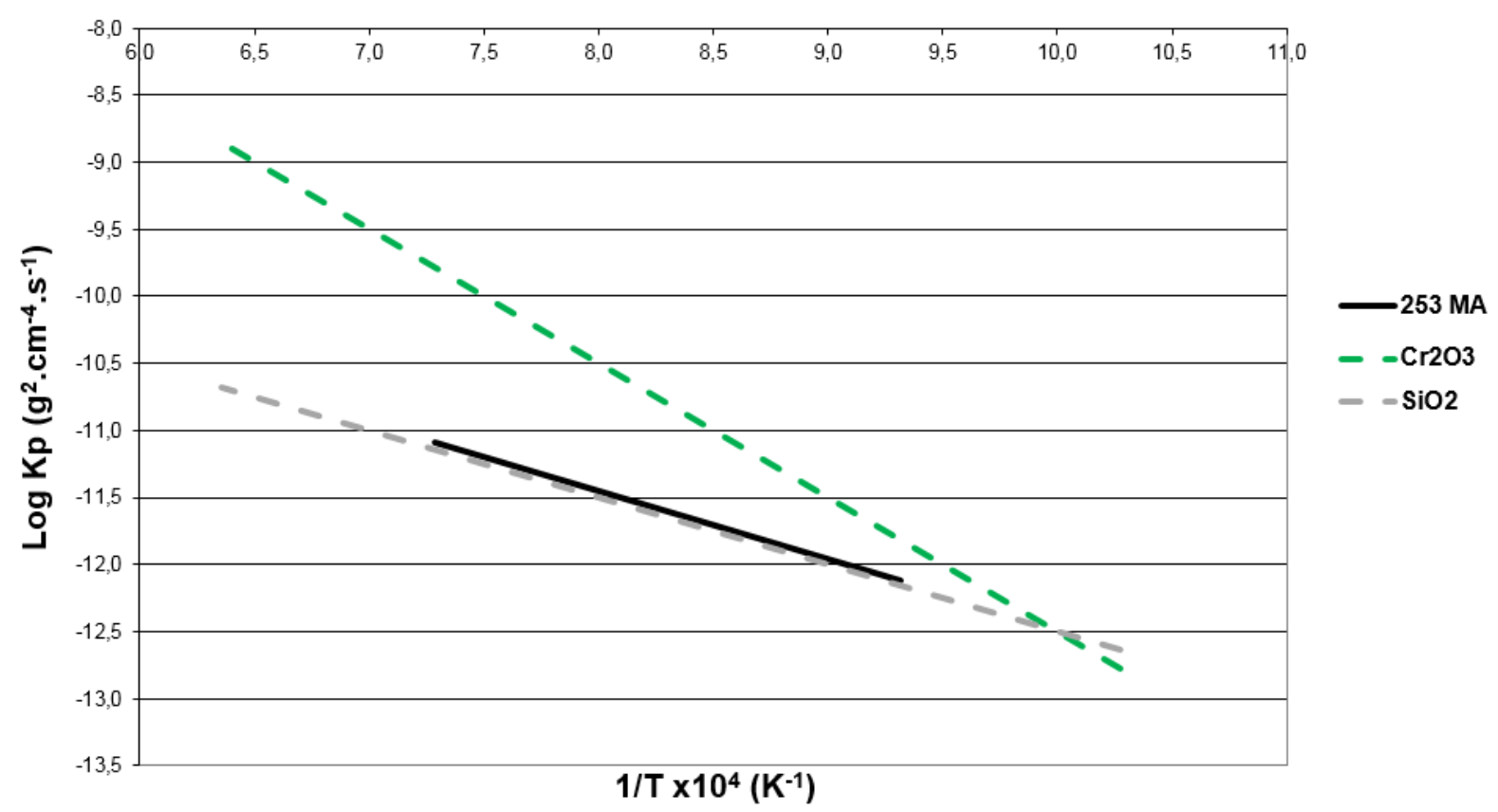

Figura 5 - Valores de log $\left(K_{p}\right)$ em função da temperatura recíproca para os óxidos $\mathrm{SiO}_{2}$ e $\mathrm{Cr}_{2} \mathrm{O}_{3} \mathrm{e}$ para as amostras estudadas.

Os comportamentos logarítmicos encontrados nos estágios iniciais de oxidação não significam que o controle do processo seja por potencial elétrico. Mostram apenas os melhores ajustes para as cinéticas observadas.

A tabela 7 apresenta a composição química do aço laminado (253 MA®).

Tabela 7 - Composição química do aço inoxidável austenítico laminado (253 MA®),

\begin{tabular}{|c|c|c|c|c|c|c|c|c|c|c|c|}
\hline & $\mathbf{C}$ & $\mathbf{S i}$ & $\mathbf{M n}$ & $\mathbf{C r}$ & $\mathbf{N i}$ & $\mathbf{N}$ & $\mathbf{C e}$ & $\mathbf{C r e q}$ & $\mathbf{N i e q}$ & $\mathbf{C r}_{\text {eq }} / \mathbf{N i}_{\text {eq }}$ & $\mathbf{C e q}$ \\
\hline 253 MA & 0,072 & 1,58 & 0,62 & 20,92 & 10,84 & 0,16 & 0,05 & 23,29 & 18,11 & 1,29 & 0,176 \\
\hline
\end{tabular}


A adição de silício e de cério contribui para a boa resistência à oxidação do 253MA. O cério reduz a taxa de crescimento dos óxidos promovendo o crescimento de uma "subcamada" contínua de sílica $\left(\mathrm{SiO}_{2}\right)$ sob a camada de cromita $\left(\mathrm{Cr}_{2} \mathrm{O}_{3}\right)$. [14] $\mathrm{O}$ silício diminui a cinética de oxidação através da formação de uma camada de sílica $\left(\mathrm{SiO}_{2}\right)$ que retarda a difusão de cátions de ferro através da camada de óxido. [15] No entanto, o silício mostrou aumentar a tendência ao desprendimento da camada de óxidos durante a oxidação cíclica. Tal efeito deletério do silício é compensado pela adição de cério. $[15,16]$

O aço inoxidável austenítico laminado $253 \mathrm{MA} \circledast$ apresenta excelente resistência à oxidação a altas temperaturas e ao desprendimento das camadas protetoras de óxidos sob condições cíclicas no ar, em comparação com AISI 310. [14]

\section{CONCLUSÃO}

A análise dos ensaios termogravimétricos do aço inoxidável austenítico laminado $253 \mathrm{MA} \circledast$ mostrou uma cinética de oxidação complexa, passando de logarítmica nos instantes iniciais para um posterior comportamento parabólico.

O comportamento logarítmico é o que apresenta os melhores valores para os coeficientes das regressões $\left(\mathrm{R}^{2}\right)$ nas etapas iniciais de crescimento das películas de óxido, o que sugere a formação de óxidos de ferro $\left(\mathrm{Fe}_{2} \mathrm{O}_{3}, \mathrm{Fe}_{3} \mathrm{O}_{4}\right.$ e $\left.\mathrm{FeO}\right)$ a uma alta taxa inicial de crescimento.

Os valores de $\mathrm{K}_{\mathrm{p}} \mathrm{s}$ encontrados neste trabalho estão de acordo com os observados na literatura quando há formação de películas protetoras de $\mathrm{Cr}_{2} \mathrm{O}_{3}$ e $\mathrm{SiO}_{2}$.

\section{Agradecimentos}

À SMT - Sandvik Materials Technology, representada pelo caro colega politécnico Fausto Camargo e pelo caro amigo Luiz Gobbis, por acreditar neste projeto e pela doação do metal base e do consumível de soldagem bem como por sempre apoiar a Escola Politécnica da Universidade de São Paulo quando existe o interesse em realizar-se estudos envolvendo ligas resistentes à corrosão.

À Prax Air representada pelos caros colegas Marcos Lobato e Jeferson Costa, por acreditar neste projeto e pela doação dos gases de proteção para a soldagem e do ar sintético para os ensaios de oxidação bem como por sempre apoiar a Escola Politécnica da Universidade de São Paulo quando existe o interesse em realizar-se estudos envolvendo gases especiais.

\section{REFERÊNCIAS}

1) BIRKS, N.; MEIER, G. H. ; PETTIT, F. S. High Temperature Corrosion, .Superalloys, Supercomposites and Superceramics, Materials Science Series, p. 439-489, 1989.

2) WASIELEWSKI, G. E. ; RAPP, R. A. High-Temperature Oxidation, The Superalloys, Vital High Temperature Gas Turbine Materials for Aerospace and Industrial Power, p. 287-316, 1972.

3) WRIGTH, I. G. High-Temperature Corrosion, Metals Handbook, 9. ed., v. 13, p. 97-103;

4) BARRET, C. A. The Effect of Variations of Cobalt Content on the Cyclic Oxidation 
Resistance of Selected Ni-Base Superalloys, Alternate Alloying for Environmental Resistance, Proceedings of the Symposium sponsored by the Corrosion and Environmental Effects Committee of The Metallurgical Society of AIME, Louisiana, $p$. 211-231, 1986.

5) SANTORELLI, R.; NORTON, J. F.; BREGANI, F. High Temperature Corrosion of Some Fe-Ni-Cr Commercial Alloys in Gaseous Environments with Relevance to Coal Conversion Processes, $11^{\text {th }}$ International Corrosion Congress. Florence, v.4, p. 4.14.10 1990;

6) DOYCHAK, J. K.; MITCHELL, T. E; SMIALEK, J. L. High Temperature Oxidation of $\beta$-NiAl, High-Temperature Ordered Intermetallic Alloys, Materials Research Society Symposium Proceedings, v. 39, p. 475-484, 1984.

7) BARNES, J. J.; SRIVASTAVA, S. K. Oxidation of Gas Turbine Seal Ring Alloys and Its Effect on Mechanical Properties. $7^{\text {th }}$ International Symposium on Superalloys. TMS, ASM, ASME, p. 825-833, 1992.

8) BRILL, U. ; KLÖWER, J. High Temperature Corrosion of Intrmetallic Phases Based on $\mathrm{Ni}_{3} \mathrm{Al}$. High-Temperature Ordered Intermetallic Alloys IV, Materials Research Society Symposium Proceedings, v. 213, p. 963-968, 1990.

9) PINT, B. A.; JAIN, A.; HOBBS, L. W. The Effect of Yttrium Ion Implantation on the High Temperature Oxidation Properties of NiAl, High-Temperature Ordered Intermetallic Alloys IV, Materials Research Society Symposium Proceedings, v. 213, p. 981-986, 1990.

10) WOLYNEC, S. Oxidação de Metais, Corrosão e Proteção Contra Corrosão de Metais. IPT 1981. (Publicação IPT No 1127), Coordenador Deniol K. Tanaka, p. 75104.

11) NARDOU, F.; RANAIVONIARIVO, L.; RAYNAUD, P.; BILLY M. Relaxation of the Mechanical Stresses Developed Through Oxide Scales During Oxidation of Metals, High Temperature Alloys-Their Exploitable Potential, Commission of The European Communities, p. 89-96.

12) MCKEE, D. W.; FLEISCHER, R. L. Oxidation Behavior of Advanced Intermetallic Compounds, High-Temperature Ordered Intermetallic Alloys IV, Materials Research Society Symposium Proceedings, v. 213, 1990, p. 969-974.

13) WASIELEWSKI, G. E. ; RAPP, R. A. High-Temperature Oxidation, The Superalloys, Vital High Temperature Gas Turbine Materials for Aerospace and Industrial Power, p. 287-316, 1972.

14) ANDERSSON, T., ODELSTAM T. - Sandvik 253MA (UNS S30815) - The Problem Solver for High Temperature Applications, A Sandvik Publication, Oct. 1984.

15) VANGELI, P., IVARSSON, B. - "Investigation of a new methodology in high temperature oxidation application to commercial austenitic steels", Materials Science Forum, vol. 369-372, 2001, pag. 785-792.

16) BASU, S. N., NATH, D., TEBBETS, J., "Microstructural evolution of multilayered oxide scales on stainless steels", in MATERIALS RESEARCH SOCIETY SYMPOSIUM PROCEEDINGS; 280; 541-544; Evolution of surface and thin film microstructure by Materials Research Society, Pittsburgh, PA; 1993. 